INPLASY

PROTOCOL

To cite: Molina et al.

Supracrestal circumferential

fibrotomy as a treatment strategy for recurrence in patients with orthodontics: a systematic review. Inplasy protocol 202130044. doi: 10.37766/inplasy2021.3.0044

Received: 15 March 2021

Published: 15 March 2021

Corresponding author:

Edith Molina

edithmolinamiranda@gmail.com

Author Affiliation:

Department of Periodontology, School of Dentistry, Universidad Científica del Sur, Lima, Perú.

Support: Self-financing.

Review Stage at time of this submission: Data extraction.

Conflicts of interest:

None declared.

\section{Supracrestal circumferential} fibrotomy as a treatment strategy for recurrence in patients with orthodontics: a systematic review

Molina, E1; Torrejón, S2; Mendoza, G33; Vernal, X4; Cafferata, EA5.

Review question / Objective: To determine the efficacy of the circumferential supracrestal fibrotomy technique to reduce the recurrence of orthodontically treated rotated teeth.

Condition being studied: Orthodontic recurrence evaluated in degrees $\left(^{\circ}\right)$ in study models.

Information sources: The search will be performed in the MEDLINE via Pubmed, EMBASE, Cochrane's CENTRAL, Scopus, Web of Science's, clinicaltrials.gov and LILACS electronic databases using search strategies (Supplemental $X)$. In addition, the grey literature databases, OpenGrey and PQDT-ProQuest, will be searched for potential studies. Moreover, a complemental manual search will be performed in the following orthodontics journals: European Journal of Orthodontics, American Journal of Orthodontics and Dentofacial Orthopedics, Angle Orthodontist, Journal of Clinical Periodontology, Journal of Periodontology, and International Journal of Periodontics and Restorative Dentistry. Finally, the references of the retrieved studies and similar reviews will be also revised.

INPLASY registration number: This protocol was registered with the International Platform of Registered Systematic Review and Meta-Analysis Protocols (INPLASY) on 15 March 2021 and was last updated on 15 March 2021 (registration number INPLASY202130044).

\section{INTRODUCTION}

Review question / Objective: To determine the efficacy of the circumferential supracrestal fibrotomy technique to reduce the recurrence of orthodontically treated rotated teeth.
Rationale: This research will provide up-todate and systematic information on the efficacy of supracrestal circumferential fibrotomy in reducing recurrence of orthodontically treated rotated teeth. The importance of this study lies in the difficulty 
of predicting the recurrence of rotated teeth due to different factors that affect the stability of the results of orthodontic treatment such as the musculature of the mouth, skeletal differences that are not adequately corrected, migration normal physiological and periodontal factors. Likewise, it should be noted that the lack of knowledge and experience on this technique by orthodontists and periodontists means that it is not considered as a common treatment in this type of cases, being able to provide beneficial effects to the patient, guaranteeing a decrease in recurrence and achieve successful long-term treatments.

Condition being studied: Orthodontic recurrence evaluated in degrees $\left(^{\circ}\right)$ in study models.

\section{METHODS}

Participant or population: Teeth that received orthodontic treatment.

Intervention: Supracrestal fibrotomy.

Comparator: No additional intervention.

Study designs to be included: Human studies, including randomized or nonrandomized controlled clinical trials, prospective, retrospective, and retroprospective cohort studies. There will be no language or publication status restrictions.

Eligibility criteria: In order to answer to the research question: Does supracrestal fibrotomy reduces the risk of rotation relapse in orthodontically treated teeth? Publications that met the following PICO format and inclusion criteria were included. - P: Teeth that received orthodontic treatment. - I: Supracrestal fibrotomy. - C: No additional intervention. • O: Orthodontic relapse by means of rotation angle relapse. Due to the limited amount of randomized clinical trials evaluating supracrestal fibrotomy, all human studies including randomized or non-randomized controlled trials, observational studies and case series will be considered for inclusion.
Animal studies, editorial letters, and reviews will be excluded. There will not be any language, year and publication status restrictions. Eligible publications in languages different from English, Spanish, French or Portuguese will be translated.

Information sources: The search will be performed in the MEDLINE via Pubmed, EMBASE, Cochrane's CENTRAL, Scopus, Web of Science's, clinicaltrials.gov and LILACS electronic databases using search strategies (Supplemental X). In addition, the grey literature databases, OpenGrey and PQDT-ProQuest, will be searched for potential studies. Moreover, a complemental manual search will be performed in the following orthodontics journals: European Journal of Orthodontics, American Journal of Orthodontics and Dentofacial Orthopedics, Angle Orthodontist, Journal of Clinical Periodontology, Journal of Periodontology, and International Journal of Periodontics and Restorative Dentistry. Finally, the references of the retrieved studies and similar reviews will be also revised.

Main outcome(s): Orthodontic relapse by means of rotation angle relapse.

Quality assessment / Risk of bias analysis: Clinical trials will be assessed with the Cochrane's Risk of Bias 2 (ROB-2) tool, observational studies will be assessed with the Newcastle-Ottawa (NOS) tool, and the case series will be assessed with the Institute of Health Economics Quality Appraisal (IHEQA) tool. Calibration rounds will be performed with all the tools previous to the assessment. Calibrated examiners (EM, ST) will evaluate the ROB of the included articles independently and in duplicate, if disagreements occur a third author (EAC) will be consulted.

Strategy of data synthesis: If substantial heterogeneity between studies exists, a qualitative synthesis of results will be performed. 12 or the Cochrane's $Q$ statistic will be used to assess heterogeneity. Depending on the degrees of homogeneity, a fixed-effect or random-effect metaanalysis will be chosen. Effect size 
estimates will be expressed as relative risks with a $95 \%$ confidence interval.The data analysis will be performed using the SPSS software v.22.0 (IBM Corp, Armonk, NY, USA).

Subgroup analysis: None reported.

Sensitivity analysis: None reported.

Language: There will not be any language, year and publication status restrictions. Eligible publications in languages different from English, Spanish, French or Portuguese will be translated.

Country(ies) involved: Perú.

Keywords: The search method will be carried out using the combination of keywords in the different search engines such as: rotation.

Contributions of each author:

Author 1 - Edith Molina - The author wrote the manuscript.

Author 2 - Silvia Torrejón - data selection.

Author 3 - Gerardo Mendoza - The author contributed to the development of the selection criteria, and the risk of bias assessment strategy.

Author 4 - Rolando Vernal - The author provided statistical expertise.

Author 5 - Emilio A. Cafferata - The author read, provided feedback and approved the final manuscript. 